\title{
Value Modelling for Multi-Stakeholder and Multi-Objective Optimization in Engineering Design
}

Evangelos Papageorgiou, Murat Hakki Eres and James Scanlan

Computational Engineering and Design Research Group, Faculty of Engineering and the Environment, University of Southampton, Southampton, SO16 7QF, UK

Dr Evangelos Papageorgiou, Southampton Boldrewood Innovation Campus, Building 176, University of Southampton, Burgess Road, Southampton, SO16 7QF, UK, email: ecpapage@gmail.com (corresponding author).

Dr M. Hakki Eres, Southampton Boldrewood Innovation Campus, Building 176, Room 5025, University of Southampton, Burgess Road, Southampton SO16 7QF, UK.

Professor James Scanlan, Southampton Boldrewood Innovation Campus, Building 176, Room 5027, University of Southampton, Burgess Road, Southampton SO16 7QF, UK. 


\section{Value Modelling for Multi-Stakeholder and Multi-Objective Optimization in Engineering Design}

The work presented culminates in the development of a value model used in the conceptual phase of engineering design, with the preferences of more than one stakeholder addressed in the Multi-Stakeholder and Multi-Objective Optimization. The "value" of proposed solutions is assessed in an objective way both from performance and economic perspectives, while the optimal solution is identified based on the needs of the user and manufacturer, as major stakeholders. This novel value model for consistent value assessment synthesizes the multiattribute value/utility analysis with the Game Theory and Analytic Hierarchy Process (AHP) assessment methodologies. During the multi-criteria decision analysis, the deficiencies, introduced by the arbitrary numerical scales used in AHP to convert the linguistic preferences of the user between the various attributes to numerical values, are resolved. The preferences of a group of experts/decision makers are synthesized in the group value model through the extraction of weighting factors from the individuals' AHP pairwise comparison matrices. Moreover, Game Theory is used in a hybrid cooperative/bargaining, non-cooperative non-zero sum game between the stakeholders as players, identifying the optimal design through the simultaneous employment of the Nash bargaining solution and the Nash equilibrium.

Keywords: Multi-Criteria Decision Analysis (MCDA), Analytic Hierarchy Process (AHP), Multi-attribute Utility Theory (MAUT), Nash equilibrium, Nash bargaining solution (NBS).

\section{Introduction}

In order to locate the best solution in engineering design, the optimization process should explore the widest possible design space; however, to address all economic and non-economic needs of stakeholders involved in order to identify the value-enhancing design(s), the goodness of all proposed system solutions needs to be assessed using the multiple criteria decision analysis (MCDA) approach. This paper presents 
methodologies and results of the authors' current research for the assessment of "value" of proposed systems solutions, relying on both performance and financial needs analysis, within the development of a value-driven conceptual design assessment framework. It is imperative that the value model captures the needs and requirements of all stakeholders involved during the designed system's whole lifecycle through the appropriate design attributes, framing and guiding the intricate process of engineering design. During the conceptual design phase, only basic needs and vague requirements are known and the widest possible design space is explored. Ultimately, the most preferred designs, based on their evaluation against technical and economic criteria, are selected for further analysis. Therefore, the objectivity of the evaluation has to be based only on those needs and preferences of the stakeholders involved, with criteria independent of the proposed alternative solutions. Multi-criteria decision analysis techniques are reviewed in Section 2, followed by the presentation in Section 3 of the proposed value model utilised in a conceptual value driven design optimization. An application of this value model in a case study of a small Unmanned Air System (UAS) conceptual design is described in Section 4 and finally primary contributions to the current state of knowledge and conclusions are outlined in Section 5.

\section{Literature Review}

The identification and structuring of all stakeholders' needs/requirements, covering the complete lifecycle of the designed product, is the first step of the engineering design process. As Keeney (1996) points out, these needs will pronounce the values that should be addressed with the engineering design and steer both the collection of information and decision making. Keeney and Raiffa (1976) describe the process of constructing a non-unique hierarchy of objectives, with one or more comprehensive and measurable attributes associated to each of them; constituting thus a complete, operational, 
decomposable, non-redundant, and minimal full set of attributes as the input of the objective/value function, modelling the overall goodness of a particular design alternative.

Several approaches can be used during the multi-criteria decision making process of evaluating a set of alternatives which are depicted by a number of attributes with the corresponding objectives being pursued. The maximization of value is fundamental to any decision-making problem solving; hence, economic theory reflected in cost contributes pari passu with system performance in the creation of the value model. The most significant approaches in Multi-Criteria Decision Analysis (MCDA) include ELECTRE (Roy 1991), multi-attribute utility theory (MAUT) ((Fishburn 1970), (Keeney and Raiffa 1976)), simple multi-attribute rating technique (SMART) ((Edwards 2009), (Edwards and Barron 1994)), Analytic Hierarchy Process (AHP) (Saaty 1980), Weighted Objectives Method (Roozenburg and Eekels 1995), technique for order preference by similarity to ideal solution (TOPSIS) (Yoon and Hwang 1995) and simple additive weighting (SAW) (Kirkwood and Corner 1993). Collopy (2009), surveying some of these MCDA techniques used in the development of the value model for engineering design, concluded that it is the user's point of view, adopted in value modelling, that defines the selection of the most appropriate tools. The MCDA techniques utilised in the development of the value model for the conceptual phase of engineering design are presented in the following sections.

\subsection{Multi-Attribute Utility and Value Modelling}

Among the various multiple criteria decision-making approaches, the multi-attribute utility theory (MAUT) has a prominent place, mainly due to its comprehensive theoretical structure. Its limitations lie in the fact that the goodness of design alternatives is measured through an abstract utility index and it is inappropriate when 
more than one individual is considered due to Arrow's Impossibility Theorem, (Arrow 1963).

Utility and multi-attribute utility theory, (Fishburn 1970) and (Keeney and Raiffa 1976) respectively, measure preferences of consequences with one or more dimensions, capturing the stakeholder's attitude towards uncertainty. The preferential independence and utility independence are usually assumed and the model is a multiplicative one:

$$
K \cdot U(X)+1=\prod_{i=1}^{n}\left[K \cdot K_{i} \cdot U_{i}\left(X_{i}\right)+1\right]
$$

In this equation, $U$ and $U_{i}$ are the total and individual utility functions respectively, while $K_{i}$ are the scaling constants and $K$ is an additional normalization constant generated through the iterative process presented in Appendix 6B, (Keeney and Raiffa 1976). Multi-attribute utility theory has been used as a standard MCDA technique in several applications in research and real-world problems, such as the airline industry (Chang and Yeh 2001), farming systems (Van Calker et al. 2006), nuclear energy (Kim and Song 2009) and earthquake projects (Butler, Morrice and Mullarkey 2001) to deep-space observation missions (Ross et al. 2010).

Value functions, as special cases of utility functions, ignore the stakeholder's risk attitude and represent the worth the stakeholder gives under certainty to achieve a certain value for a design attribute. The additive (preferential) independence among the attributes is assumed, yielding the following multi-attribute additive value model:

$$
V\left(X_{1}, X_{2}, \ldots, X_{n}\right)=\sum_{i=1}^{n} K_{i} \cdot V\left(X_{i}\right)
$$

The scaling constants $K_{i}$ are assessed using two approaches, the trade-off and the direct rating approaches, described by Keeney and Raiffa (1976) and Dyer and Sarin (1979) respectively. The individual value functions $V\left(X_{i}\right)$ of the attributes are computed 
independently with a direct value estimation technique, using three distinctive approaches: direct rating, direct midpoint and direct ordered metric (Fishburn 1970). Multi-attribute value models are employed when the stakeholder's risk attitude is ignored, as in the value assessment of various business airplane designs (Downen, Nightingale and Magee 2005). They were also used in conjunction with bio-economic modelling to study production strategies and the use of soil nutrient resources (Berkhout et al. 2011), and with AHP and fuzzy set based approach in the nuclear spent fuel management (Sohn, Yang and Kang 2001). Value functions were combined with utility functions, eliciting values (through linear value functions) at lower-levels of the objectives hierarchy and assessing risk attitudes at higher levels (Schuwirth, Reichert and Lienert 2012).

\subsection{Analytic Hierarchy Process}

The Analytic Hierarchy Process (AHP) is based on judgments from a group of experts or decision makers and it applies hierarchical decomposition of a high level objective to lower level sub-objectives as described by Saaty (1980). AHP uses pairwise comparisons between all possible pairs to establish an objective weighting for evaluation of a number of alternatives with respect to several criteria/attributes (Saaty and Vargas 2001). Additionally, AHP compensates for any bias or inconsistency through the redundant pairwise comparisons' construction of the judgment matrix, assessing the consistency/validity of the model.

However, AHP lacks the sound theoretical axiomatic foundation of the utility theory (Von Neumann and Morgenstern 1953), as pointed out by Dyer (1990). Also, the phenomenon of Rank Reversal could happen, with the ranking between the dominating alternatives changing, when other dominated or inferior alternatives are introduced (Perez 1995). Another disadvantage of the AHP is the construction of its matrix, which 
is based on the ambiguous question: 'How much better/more important is

attribute/alternative $A_{i}$ than $A_{j}$ ?' assessing the ratio scaled strength of preference. It is however used for defining the problem, considering a large number of attributes, communicating value, identifying differences and similarities between various stakeholders' points of view and aggregating them as presented in (Lai, Wong and Cheung 2002), (Morera 1998), (Gass 2005), (Chen 2007) and (Scholl et al. 2005). Nevertheless, it is the synthesis of MAUT with AHP that offers significant benefits in the preferences' assessment, as discussed by Dyer (1990) and corroborated by several applications, such as (Poyhonen 2001), (Sohn, Yang and Kang 2001), (Crossman 2011), (Abu Dabous 2010), (Sha and Che 2005) and (Tarek and Elsayed 2013).

\subsection{Group Decision Making}

Multi-attribute utility theory elicits the preferences of an individual decision maker, assessing the entire set of alternatives by developing and adding up appropriate utility functions for all attributes. In most cases, several individuals/experts constitute a decision group and an appropriate aggregation method is required to obtain the corresponding group objective function, while the conflict between the preferences of the group members is a common situation.

MCDA methods provide useful tools to deal with interpersonal preferential conflicts, aiming to achieve group members' consensus. AHP is well equipped for group decision making (Dyer and Forman 1992) through consensus, voting/compromising, computing the geometric/arithmetic mean of individuals' judgments, or any other way of averaging the individual results. Several applications of this aggregation are presented in literature, such as the computation of the arithmetic mean of the individuals' preferences after being normalized appropriately in 
(Matsatsinis, Grigoroudis and Samaras 2005) and (Feng and Lai 2013). Alternatively, the geometric mean is also used in AHP and fuzzy AHP techniques (Lai, Wong and Cheung 2002), (Kim and Song 2009), and (Carnero 2013). In an ordinal ranking, the individuals' preferences can also be aggregated (Hurley and Lior 2002) and quite often the preferential differences among different alternatives and priorities are also considered/weighted to obtain the group utility values (Huang et al. 2013). Dijkstra (2013) presented a method for the extraction of group weighting factors from the group members' AHP pairwise comparison matrices, minimizing the inconsistencies introduced in the group preference synthesizing. Nevertheless, the selection of the averaging method used in the aggregation of the individuals' preferences is rather arbitrary, unless it explicitly deals with the interpersonal comparison of preferences.

\subsection{Application of Game Theory in Engineering Design}

In engineering design, several decisions have to be taken into account concerning the whole lifecycle of the designed system, including the design, manufacturing, use, maintenance, repair and disposal stages. These decisions made by different stakeholders can be studied using the Game Theory, since all $N(>1)$ stakeholders as players aim to better promote their interests, through the maximization of their own objective function, while they are affected by the others' choices.

Although John Von Neumann is considered to be the founder of the Theory of Games (Von Neumann and Morgenstern 1953), the keystone was set by John Nash with his proposed Nash equilibrium solution of non-cooperative games (Nash 1950) and Pareto optimal Nash bargaining solution (NBS) of cooperative games (Nash 1950). The Nash equilibrium constitutes the set of all players' strategic choices and their corresponding payoffs, if each player has chosen a strategy and no player can benefit by changing their strategy, while the other players keep theirs unchanged. Game Theory as 
an optimisation tool, modelling decision interactions among rational players as noncooperative games, has been applied in numerous engineering design cases. Nash equilibrium was employed in the engineering asset management between maintenance chain participants in a negotiation model (Trappey, Trappey and Ni 2013), between engineering disciplinary teams for collaborative decision making (Xiao et al. 2005), and the design of an aero-structural aircraft wing shape optimisation (Desideri 2012). The selection of players varies from actual persons, agents to aircraft components evaluated (Runyan et al. 2010) or using different disciplines/technologies (Habbal, Petersson and Thellner 2004). It was also applied based on gene expression programming in multiobjective multidisciplinary design optimization (MDO) problems (Xiao 2015). Players can be fictitious, each having control of one design variable in a particle swarm optimisation (Annamdas and Rao 2009), or even objective functions in a bargaining multi-objective optimal design (Gonzalez et al. 2007) and (Hu and Rao 2009). A hybrid-game strategy for multi-objective design optimization was proposed by Lee $e t$ al. (Lee 2011), employing Nash equilibrium as a fast companion optimizer to guide the slower multi-objective evolutionary optimizer, capturing the Pareto non-dominated front.

Nash equilibrium of games between non-cooperative players does not guarantee the property of Pareto Optimality of the solution obtained. However, if players cooperate through a bargaining process, they are rewarded with a solution that belongs to the Pareto optimal set. The problem of indeterminacy of the Pareto front was solved by Nash (1950) through the determination of a definite solution among all the Pareto optimal candidates, representing the anticipations the players would agree upon as fair bargains. It is based on the criterion of maximization of the product of utilities' distances from the disagreement points for the bargaining problem between two players, 
reaching a binding agreement. Harsanyi and Selten (1972) generalized the bargaining problem for two or more players of not equal relative authorities. The NBS has been identified in several cases modelled by cooperative games, such as the design and management of microwave access networks (Jiao et al. 2011), design of controllers in a multi-agent team cooperation approach (Semsar-Kazerooni and Khorasani 2009), bandwidth allocation in high-speed networks (Yaiche, Mazumdar and Roseberg 2000), (Ma et al. 2012), design of water distribution networks, (Beygi 2014), and collaborative product development (Arsenyan, Buyukozkan and Feyzioglu 2015).

\section{Multi-Stakeholder Multi-Objective Value Model}

In engineering design, two distinctive features are involved, the complexity/uncertainty analysis required to develop and validate the design generative model, and the preference analysis to capture the values of the stakeholders in the value model. As Keeney and Raiffa (1976) point out, the engineering design practice is clearly in favour of the alternatives' generation modelling and against the preferences/value modelling.

In the proposed value model, an array of attributes which is based on the objectives of all involved stakeholders reflecting performance, economic and other concerns and measured in incommensurable units, models the relative goodness of any future design. These attributes are measured in incommensurable units and they cannot be collated into an objective function. Hence, the multi-attribute multiplicative utility model of equation (1) and the additive value model of equation (2) were developed to assess the stakeholders' preferences. The multiplicative utility model was created based on standard MAUT, following the methodology presented in (Keeney and Raiffa 1976). This model is more complicated and elaborate to develop than the value model, since it requires more interaction with the stakeholders, in order to assess their risk attitude and obtain the utility functions. However, the effectiveness of MAUT in capturing the 
preferences of the stakeholder has been validated and it has been used as a standard technique in both research and real-world problems, as already discussed in section 2.1. Hence, this multi-attribute utility model is utilised as a means of validating the proposed additive value model.

The multi-stakeholder, multi-objective value model, assessing the stakeholders' preferences during the conceptual design phase, is based on MAUT, AHP and Game Theory. MAUT is employed in the multiple objectives optimization due to its theoretical axiomatic foundation, while Game Theory is used to address the preferences of multiple stakeholders in engineering conceptual design. The aggregation of individual preferences is performed through a synthesization averaging AHP-based method.

The assignment of average levels of expectations with respect to the attributes by the stakeholder is the basis of this additive value model, used for both the scaling constants $K_{i}$ and value functions $V_{i}$ assessments. Thus, as Keeney (1996) advocates, the alternative-focused process of selecting the best from what is readily available, is converted to a value-focused process of identifying needs, attributes and values of these attributes that give the user a 'neutral' response, a 50\% satisfaction level, described by Eres et al. (2014) in the Concept Design Analysis (CODA) methodology. The major advantage of this value model is that it is an efficient and operational way to evaluate each design point with minimum interaction with the stakeholder during the conceptual phase, when only basic needs and vague requirements are known and the design is not finalized. The objectivity of the evaluation is maintained, capturing the stakeholder's preferences with criteria independent of information, other available data or the proposed alternative solutions. The advantages and disadvantages of the proposed value model compared to the multi-attribute utility model based on standard MAUT are 
presented in Table 1. The constituent parts of this model are presented in the following subsections.

Table 1 Advantages/Disadvantages of Value/Utility Models Near Here

\subsection{Value Functions}

Before the conceptual design space exploration starts (subject to the technology readiness level assumed), neutral values for all attributes are provided $a$ priori by the stakeholder. Next, the relationship type for each attribute is defined, i.e. maximizing, if more is better (for performance attributes), minimizing, if less is better (for cost related attributes) or optimizing, if a specific attribute value is better. The preferences of the stakeholder are qualitatively assessed in terms of the marginal evaluation with respect to each attribute, i.e. how much they are willing to sacrifice in terms of other attributes for a positive change of this attribute as its value changes, reflected in the slope of the value functions. The attributes' value functions are thus adjusted as concave, convex or sigmoidal functions. A convex value function reflects the preferences of a stakeholder who is willing to sacrifice more and more in terms of other attributes for the same positive increment as this attribute's values increase. A concave value function is selected if they are willing to sacrifice less and less in terms of other attributes for the same positive increment as this attribute's values increase. A sigmoidal shape is selected for mixed preferences, i.e. if they are willing to sacrifice more and more in terms of other attributes for the same positive increment of this attribute up to the neutral point/inflection point and less and less beyond that.

Depending on the previous selections, the appropriate, ready to use, value function is automatically generated from all available, presented in Table 2, with the minimal interaction with the stakeholder. In this Table, $X$ is the input attribute value for 
any design, $n$ is the assigned neutral value of the design attribute, while $V_{i n}$ and $V_{f}$ are the initial and final values of the value functions (set accordingly 0 and 1 , depending on their type).

Table 2 Value Functions Near Here

\subsection{Assessment of Weighting Factors}

Next, AHP is employed to perform pairwise comparisons between the attributes and not only provide the values of the weighting factors, but also assess the consistency of the answers provided by the stakeholder. However, as stated before, the construction of the AHP matrix is based on the ambiguous question: 'How much better/more important is attribute/alternative $A_{i}$ than $A_{j}$ ?' which assesses the ratio scaled strength of preference. It has been found that this unjustifiable selection of numerical scale, such as those presented by Elliot (2010), converting the linguistic response to the above question to ratio scaled numerical values, greatly affects the identification of optimal design.

The following AHP numerical scales convert the stimuli/psychological perception of strength of preference increments among various attributes to a response/numerical value through the use of an arbitrary relation. The integer scale assumes a logarithmic relation between stimuli and response; it was used by Saaty (2000) to obtain the integer sequence $1,2,3, \ldots, 9$ of the integer scale in AHP. The balanced scale assumes an even distribution of attribute weights, (Salo and Hamalainen 1997), obtaining the following scale: $1, \frac{11}{9}, 1.5, \frac{13}{7}, \frac{7}{3}, 3,4, \frac{17}{3}, 9$. Whilst the power scale assumes a geometric relation between stimuli/psychological perception of strength of preference increments among various attributes and response/numerical values (Lootsma 1991). Hence, for a $1-9$ numerical scale and nine increments, the power scale sequence is $1,1.316,1.732,2.28,3,3.948,5.196,6.84,9$. 
As presented in Table 3 for integer and power scale and five degree of preference scheme, they quantify exactly the same linguistic responses to different numerical values. For instance the verbal response: ' $A_{i}$ is much more important than attribute $A_{j}^{\prime}$ is converted to a numerical value of 7 with integer scale and to a value of 5.2 with power scale, with the integer scale favouring more the most important to the user attributes and weighing less the least important ones.

\section{Table 3 AHP Numerical Scales Near Here}

Due to the absence of justified criteria for selecting a particular AHP numerical scale, the distribution of weights and the level of consistency obtained by these scales were studied to compare them. These distributions of attribute weights obtainable by the three scales, integer, balanced and power scale, are presented for comparisons (in this order) in Fig. 1a, 1b and 1c, for a nine degree preference scheme between just three attributes, similar to Fig. 1 presented by Elliot (2010). In these figures, the $\mathrm{x}$ and $\mathrm{y}$ coordinates of each plotted point are the values of the weights of the two of the three attributes. These were obtained with a full factorial Design of Experiments (DoE) computer algorithm from all possible sets of linguistic preferential judgments, using the specific numerical scale in the AHP matrix. The third attribute's weight is computed simply by subtracting the sum of weights of the two others from one, since the sum of their weights is always equal to unity. Thus, the three scales can be compared in terms of the number of distributed points and existence of sparse regions, in order to select the scale with the highest number of points and the least sparse regions in these distributions.

Figures 1a, 1b, 1c Weights Distributions of AHP Scales Near Here

Elliot (2010) identifies the power scale as the most preferable scale; however, in Fig. $1 \mathrm{a}, 1 \mathrm{~b}$ and $1 \mathrm{c}$ the following are noticed: 
- The integer scale gives a high number of points in the extreme values of weights as expected, considering the distribution of values of the integer scale, 1, 2, 3, 4, $5,6,7,8,9$, that weighs more the most important to the stakeholder attributes and less the least important ones. Due to the distribution of values of the balanced and power scales, 1, 11/9, 4/3, 13/7, 7/3, 3, 4, 17/3, 9 and 1, 1.316, 1.732, 2.28, 3, $3.948,5.196,6.84,9$, respectively, these scales are more evenly distributed than the integer scale.

- Comparing the sparse regions and clustering obtained with these scales; the power scale fails to cover a larger area in the graph than the integer and balanced scales, which produce a definitely higher number of points and less clustering. This observation is not in agreement with Elliot (2010), who reported larger sparse regions and clustering of the weights in integer and balanced scales.

Furthermore, to demonstrate this AHP deficiency, in the multi-disciplinary optimization using exactly the same verbal responses/preferences provided by the user of the UAS and converting them into numerical weights through different AHP scales, produced different optimal design alternatives. In the UAS conceptual design and identical user's preferences with the integer scale a V-shape tail, push propeller, conventional fuselage with a wingspan $1.5 \mathrm{~m}$ aircraft was identified as optimal; while through the power scale, an aircraft of T-shape tail, pull propeller, conventional fuselage with a wingspan of $1.25 \mathrm{~m}$ was the optimal solution.

The problem of converting verbal preference responses between abstract attributes to numerical values through the use of some arbitrary scales in AHP can be tackled, if the user is forced to compare specific value differences of these attributes. The theory of measurable multi-attribute value functions, presented by Dyer and Sarin (1979), is applied to assess the strength of preferences (value differences) between 
alternatives. In the AHP matrix as the one for the UAS conceptual design (See Table 4), instead of comparing abstract attributes, each cell is the ratio of relative importance/preference of a change from 0 to the neutral value of the row attribute to the change from 0 to the neutral value of the column attribute. Following the methodology of AHP, for $n$ attributes $\left(\begin{array}{l}n \\ 2\end{array}\right)=\frac{n \cdot(n-1)}{2}$ redundant pairwise comparisons are performed, not only to compute the values of the weighting factors, but to assess also the consistency of the answers provided by the stakeholder. The values in each cell of the AHP matrix, given by the stakeholder/expert, do not represent the precise values but are mere estimates of the actual ratios; hence, the consistency ratio computed in AHP is used to assess their goodness.

Table 4 AHP Weighting Factors Assessment Near Here

In the proposed multi-attribute value model, based on the qualitative assessment of the stakeholder's preferences and the quantitative assignment of neutral values of attributes, all design alternatives are objectively evaluated during the conceptual phase, independent of information, with the minimum interaction with the user. AHP is implemented in the weighting factors' computation to increase the accuracy and to assess the consistency of the stakeholders' responses. The deficiency of converting verbal preference responses between attributes to numerical values, using an arbitrary scale in AHP for the calculation of weighting factors, is tackled by constraining the decision maker to assess strength of preferences between design alternatives, instead of comparing abstract attributes.

\subsection{Aggregation of Individual Preferences}

For the purposes of engineering design, a group of knowledgeable experts/individuals involved throughout the whole lifecycle of the system designed constitutes a group of 
decision makers, whose preferences need to be incorporated into the user's objective function. As already discussed, in most cases the selection of the averaging method used in the aggregation of the individuals' different quantitative preferences is rather arbitrary. Moreover, during the conceptual engineering design the set of alternatives is not finalized for the group ranking to be obtained, identifying the optimal design among all candidates. The aggregation of individual preferences aims mostly at synthesizing the judgments/preferences of group members in value modelling, rather than averaging the individuals' rankings of a final set of design alternatives.

AHP comparison matrices provide not only an objective weighting to assess the set of alternatives, but also a measurement of consistency of the redundant answers provided by each individual. In the group value model, the judgments of $n$ experts are synthesized to obtain the group weighting factors, satisfying the requirement that the synthesis of consistent judgments ought to be consistent as well. As presented by Dijkstra (2013), for two experts with AHP comparison matrices $A=\left(a_{i j}\right), B=\left(b_{i j}\right)$, the synthesis matrix will be defined as $C=\sum(A, B):=\left(\sigma\left(a_{i j}, b_{i j}\right)\right)$, while the synthesizing function $\sigma: \mathbb{R}_{+} \times \mathbb{R}_{+} \rightarrow \mathbb{R}_{+}$, with $\mathbb{R}_{+}$the set of positive real numbers. If $\mathrm{A}$, $\mathrm{B}$ are consistent matrices, with AHP consistency defined as: $\left(a_{i k} \cdot a_{k j}=a_{i j}\right)$, then the synthesis matrix $\mathrm{C}$ should be consistent as well: $\sigma\left(a_{i k}, b_{i k}\right) \cdot \sigma\left(a_{k j}, b_{k j}\right)=\sigma\left(a_{i j}, b_{i j}\right)$.

It is proven that the synthesis of the cells of the two AHP matrices of the experts is found as, (Dijkstra 2013):

$$
\sigma(a, b)=a^{\theta} \cdot b^{1-\theta}
$$

With $\theta \in[0,1]$, set to $1 / 2$ for experts of equal power, or appropriately reflecting power and experience/competence between them. This equation can analogously be extended to an arbitrary number of experts. Based on equation (3), once the AHP matrices of 
judgments between the design attributes for all group members are obtained, a new synthesized AHP matrix is computed, with cells equal to the geometric means of the corresponding cells of the judgment matrices. In this group matrix, the weighting factors are obtained while consistency is maintained. Finally, the individual preferences in terms of the attributes' neutral points are also synthesized in the group value model by computing their arithmetic mean.

\subsection{Use of Game Theory in Value Modelling}

In engineering design, several stakeholders have different interests/stakes in and are influenced by or could influence any part of the whole lifecycle of the designed product, from the initial steps of the conceptual design up to its disposal. The optimal design should be selected as the solution giving the amount of satisfaction every rational stakeholder anticipates and agrees upon as fair bargain. This binding agreement, satisfying the rational expectations of gain of all stakeholders, would be the outcome of a bargaining process modelled by the Game Theory as a cooperative $n$ players' non-zero sum game. This Bargaining Problem is solved in an axiomatic way with the NBS. Moreover, in the pursuit of values articulated by the stakeholders' objectives, each stakeholder is forced to make decisions in isolation, influencing the delivery of value to all others. Each stakeholder's objectives are modelled by an objective function and the decisions made are considered as strategies, promoting these interests through the maximization of this objective function. The interaction of stakeholders' strategic choices and the corresponding payoffs can be studied as an $n$ players' non-zero sum, non-cooperative game, solved through Nash equilibrium.

Several stakeholders can be selected as players in the application of the Game Theory in engineering design, for brevity the manufacturer and user of the system were chosen as the most typical players. However, this game can be extended to include more 
stakeholders as players, such as suppliers, public/local authorities, and so on. For the user, the group multi-attribute value function already presented is considered as the objective/payoff function. Concerning the manufacturer, a cost plus fee contract type was assumed and the corresponding objective/payoff function is modelled as a linear function of the Total Program Cost for entire lifecycle of the designed product. The basic assumptions for these players are that they are instrumentally rational, acting according to their preferences, they share Common Knowledge of Rationality (CKR), they have Common Priors or Consistently Aligned Beliefs (CAB) and they share common knowledge of the game rules (Hargreaves Heap and Varoufakis 2004). For simplicity, they are also assumed equal in bargaining skills.

This novel hybrid game, modelling the interactions between the stakeholders' preferences and their strategic choices, is created to evaluate the alternative designs. For each combination of strategic choices, the corresponding cooperative game is employed to identify the NBS, and among all bargaining solutions, the non-cooperative game identifies through Nash equilibrium the design alternative along with the combination of strategic choices, as the overall optimal solution of the game. If indeterminacy arises and multiple Nash equilibria are obtained, Nash's product of the payoff/utility functions, as proposed by Harsanyi (1995), is the sole criterion utilised for the selection of the specific Nash equilibrium solution of the non-cooperative game. Through the simultaneous employment of two players' cooperative non-zero sum games and two players' non-cooperative non-zero sum game, not only the optimum design is identified based on the user's and manufacturer's needs but their most significant strategic choices are also determined.

Modelling the engineering design process through this hybrid game is considered more effective than the pure cooperative or non-cooperative game models. 
This novel simultaneous employment of the cooperative and non-cooperative games offers the benefits of both approaches:

- It addresses the stakeholders' preferences in a functional, outcome-focused way, resolving the high indeterminacy of design alternatives through cooperative games.

- At the same time, it models the stakeholders' interactions for some important strategic choices with a process-focused non-cooperative game.

Thus, this game yields a single optimal solution, identified as both Nash equilibrium and NBS, capturing effectively both the conflict and cooperation between the stakeholders through this well-defined hybrid mathematical model.

\section{Cooperative non-zero sum bargaining game}

Nash's bargaining model is used to identify the optimum design from the Pareto front of the design alternatives' set. In general, a utility vector $\vec{u}:=\left(u_{1}, u_{2}, u_{3}, \ldots, u_{n}\right)$, with $n$ utility/payoff functions of $n$ stakeholders, characterizes each design alternative. The NBS, defined as $a^{*}$, achieves a unique bargaining solution satisfying the axioms of Pareto optimality, independence of irrelevant alternatives, independence of linear transformations and symmetry. In general, the $a^{*}$ NBS is obtained by solving the following maximization problem:

$$
\begin{aligned}
& \left(u_{1}\left(a^{*}\right)-u_{1}(\bar{a})\right) \cdot\left(u_{2}\left(a^{*}\right)-u(\bar{a})\right) \cdot \ldots \cdot\left(u_{n}\left(a^{*}\right)-u(\bar{a})\right)=\max _{a \in A}\left[\left(u_{1}(a)-\right.\right. \\
& \left.\left.u_{1}(\bar{a})\right) \cdot\left(u_{2}(a)-u_{2}(\bar{a})\right) \cdot \ldots \cdot\left(u_{n}\left(a^{*}\right)-u(\bar{a})\right)\right]
\end{aligned}
$$

In this equation, $\bar{a}$ is the disagreement point if no agreement is reached, with the corresponding values of payoff functions assumed for this case to be 0 . In the conceptual stage of engineering design, the product of the user's and manufacturer's 
utilities was used to determine the quality of each design alternative. For presentation purposes and based on the specific stakeholders' preferences, the values of the user's and manufacturer's payoff functions were plotted for the set of all available UAS alternatives in Fig. 2, forming a Pareto front of dominating designs. In this cooperative game, more players can be added of not equal relative authorities using the generalized Nash bargaining solution : $\left(v_{1}\left(a^{*}\right)-v_{1}(\bar{a})\right)^{\gamma_{1}} \cdot\left(v_{2}\left(a^{*}\right)-v_{2}(\bar{a})\right)^{\gamma_{2}} \cdot \ldots$. $\left.\left(v_{n}\left(a^{*}\right)-v_{n}(\bar{a})\right)^{\gamma_{n}}\right),($ Harsanyi and Selten 1972).

Figure 2 Payoff Functions Plot Near Here

\section{Non-cooperative non-zero sum game}

Concerning the user's and manufacturer's strategic choices, modelled by a two players' non-zero sum, non-cooperative, complete information, static game, the available list is rather long: different performance requirements, assumed constant in the design space exploration, improvement of technology, different quality control processes, and so on. For demonstration purposes, the following strategic choices were selected to define the non-cooperative game to be played:

- Application by the manufacturer of a reliability improvement program increasing the Mean Time to Failure (MTTF), modelled by the Weibull distribution parameter $\eta$, resulting to less aircraft losses and less scheduled replacements, but consequently higher acquisition and maintenance costs. This strategic choice relies on previous historic data to quantify the cost-reliability relationship (Alexander 1988). Although for most reliability improvement programs this relationship is quantified with considerable variability, significant reliability improvement is possible; but the greater the improvement the more costly the necessary investment is. 
- Selection between two different scheduled replacement/maintenance scenarios, one critical components replacement policy and one designed system replacement policy. In the first scenario, the system's critical components average replacement times, system's survival rates and lifecycle costs are computed through Monte Carlo simulations during the whole program duration. In the second scenario of a whole system replacement policy at specific intervals (in operating hours), the lifecycle cost due to reliability related losses and scheduled replacements is calculated through Monte Carlo simulations.

The above strategic choices define the non-cooperative game to be played by the players, based on all possible combinations between the strategic decisions made by them. The selection of the specific strategic choice for any of the two stakeholders depends not only on the value of the payoff function but also on the expectations of the player concerning the other player's most likely strategy.

\section{Multi-Stakeholder Multi-Objective Value Model Application in UAS Conceptual Design}

The value driven optimization process presented by Collopy and Hollingsworth (2011) is performed for the case of a small Unmanned Air System (UAS) conceptual design. The user's objectives with their corresponding attributes were first identified and structured in the hierarchy presented in Fig. 3.

Figure 3 UAS Objectives/Attributes Hierarchy Near Here

In the conceptual value driven design framework, the design variables (such as wingspan, wing aspect ratio, battery capacity, fin aspect ratio, etc.) are adjusted to generate feasible design points in the Define phase of the cycle. The extensive system attributes of Fig. 3 are calculated in the Analyse phase, and they are used as inputs into 
the value model during the Evaluate phase. The process carries on in the Search phase, through an optimization algorithm or the generation of more design points as presented in the value driven design cycle of Fig. 4.

Figure 4 Value Driven Design Cycle Near Here

Hence, the implementation process of value driven conceptual design in the Multi-Objective, Multi-Stakeholder engineering design framework, also presented in Fig. 5, was the following:

1. Identify all stakeholders involved with the designed system during its lifecycle.

2. For all stakeholders, identify their objectives/needs and associated attributes, creating their objectives/attributes hierarchy as in Fig. 3.

3. Form the multi-attribute value models, representing the objective functions of all stakeholders, to be used for evaluating the alternative designs in Evaluate phase of the Value Driven Design cycle. The development of the value/utility models was consisted of the following steps, according to the guidelines described by Keeney and Raiffa (1976):

- Preparing for assessment and familiarization, i.e. verifying/identifying the objectives/attributes of Figure 3.

- Verifying/assuming, the validity of the appropriate independence conditions.

- Identifying through interrogation appropriate qualitative characteristics of the utility/value functions, such as monotonicity, marginal evaluation, risk attitudes etc.

- Specifying quantitative restrictions, i.e. norm value of each attribute, assessing weighting factors from AHP matrices (see Table 4) etc. 
- Choosing the most suitable utility functions for the multi-attribute utility model and checking for consistency of all selections.

4. Select the appropriate design variables, the stakeholders' strategic choices and their ranges of values, used to search the design space in the Search phase.

5. Form appropriate models, for the product definition in the Define phase.

6. Develop predictive Monte Carlo simulation models, for the assessment of all attributes in the Analysis phase.

7. Integrate all models in the design tool for the multi-objective, multidisciplinary optimization.

8. Perform multi-objective optimization (MOO) and trade studies with the user of the system as the only stakeholder.

9. Form the hybrid cooperative/non-cooperative game among all players/stakeholders for multi-stakeholder optimization.

10. Generate a single optimal solution.

Figure 5 Value Driven Design Implementation Process Near Here

In the conceptual design phase, the widest possible design space needs to be explored meticulously without setting any constraints, as advocated by the Value Driven Design philosophy (Collopy and Hollingsworth 2011). However, for multivariable optimization trade studies, as the number of design variables and their ranges increase, the workload increases exponentially. For instance, to run a full factorial Design of Experiments (DoE) with six design variables and three levels for each of them, the number of design alternatives will be $3^{6}=729$, a number to be multiplied by the number of different aircraft configurations. Several design variables were selected to be varied along with their corresponding (continuous) ranges based on standard values as described in (Papageorgiou, Eres and Scanlan 2015); while the other 
design parameters were set to reasonable values. Beyond the selected design variables, in order to extend even further the design space, strategic choices of the stakeholders were also encompassed in the Game Theory application as additional, higher level variables that would normally be assumed constant throughout the MDO. All models were integrated in Isight workflow execution environment where the Designs of Experiments (DoE) and the MDO were performed. The DoE were full factorial with three levels for each variable, allowing all possible interactions to be evaluated, as well as Latin Hypercube for more random combinations' generation, with varying number of experiments. Following the DoE, the pattern-search (not gradient) method of HookeJeeves Direct Search was selected for the optimization, since it is suitable for covering wide ranges of non-linear design spaces and long running simulations. Some of the DoE and MDO results are presented in the following sections.

\subsection{Optimizing for User's Objectives}

The design space optimization aims at either maximizing the user's value or utility index, depending on which model is used (the additive value model or the multiplicative utility model). The preferences/priorities of the user, as reflected in the value model, are critical for the identification of the optimal design and can indeed provide different results.

Depending on the user's priorities between the performance and cost related attributes, different optimal designs are obtained. For example, for a user with balanced priorities, between performance (endurance, range) and lifecycle cost (acquisition and through-life), and for another user, focusing mostly on maximizing survivability, minimizing detectability and maximizing data collection capabilities, different aircraft optimal designs were obtained. For the first user, it was found that the monolithic fuselage, V-shape tail, push propeller with a wing span of $1.5 \mathrm{~m}$ configuration is 
dominating, while for the second user, the optimal UAS configuration was the monolithic fuselage, T-shape tail, push propeller with a wing span of $1.25 \mathrm{~m}$ configuration. Hence, it was verified that the incorporation of the largest possible number of different UAS configurations is essential to the successful optimization based on the user's varying preferences.

Dominant configurations/geometries maximizing value or utility index are identified in the UAS design, as presented in Fig. 6. In this figure, the maximum values of value index achieved with all configurations along with the corresponding values of utility index are plotted. The differences observed in the numerical results between the value and utility indices are caused by the different multiplicative utility and additive value models used. It is noted that, apart from some minor differences, the same trends are observed and the value model is in close agreement with the utility model. For the specific user's preferences, the monolithic fuselage, V-shape tail pusher propeller configuration, followed by the monolithic fuselage, Y-shape tail, pusher propeller configuration and monolithic fuselage, T-shape tail, pusher propeller configuration were dominating in terms of both value and utility indices. Moreover, to demonstrate group decision-making process, the preferences of the two aforementioned experts with different priorities were aggregated to obtain a synthesized group AHP matrix from the experts' AHP matrices. The optimal design in this case was a UAV with Y-shaped tail, pusher propeller, wingspan of $1.5 \mathrm{~m}$, and wing aspect ratio of 12 .

Figure 6 Comparison of UAS Configurations Near Here

Additionally, once the dominant UAS configuration was identified, the optimum range of design variables was obtained with surface plots, as in Fig. 7 and Fig. 8, demonstrating the effect of design variables or other parameters on the response (value and utility indices). In these figures, surface plots of value and utility indices versus 
wing span and wing aspect ratio are presented. All value surface plots were found to be in close agreement with the corresponding utility surface plots.

Both value and utility models pointed to selecting the same ranges of design variables, which for the specific user's preferences were a high wing aspect ratio of $11.5-12$, a wingspan of around $1.5 \mathrm{~m}$, a maximum battery capacity of $9.5-10 \mathrm{Ahr}$, and large scheduled components replacement intervals. The optimal ranges of some design variables with small influence, as the fin aspect ratio and horizontal tail aspect ratio, were not clear with the value model suggesting a horizontal tail aspect ratio of around 3.5 and the utility model an aspect ratio of around 4.5.

Figure 7 Value Surface Plot and Figure 8 Utility Surface Plot Near Here

The DoE provided estimates of sensitivity analyses of the design parameters and their effect on the response, optimised in each case. The normalised percentage effect of the design variables on the response, in this case the value index, is presented in Fig. 9. Thus the most significant parameters were identified, showing the percent effect on the response, with colour coding depending on whether that is positive (in blue colour) or negative (in red colour). It may be noticed in this figure that the battery capacity, followed by the wing AR and wingspan are the most significant variables.

Figure 9 Sensitivity Analysis Near Here

Finally, engineering design was also optimised for some critical (performance or cost related) attribute, such as operational surveillance time or acquisition cost for the UAS. It was found that the twin boom inverted V-shape tail with tractor propeller configuration with wing aspect ratio of 12 , wingspan $1.25 \mathrm{~m}$, fin aspect ratio of 1.4 and horizontal tail aspect ratio of 3.5 was the optimal design both for maximizing operational surveillance time when flying at design speed and for minimizing total lifecycle cost. 
Based on the results obtained, the benefit and effectiveness of the multi-attribute value model was verified, since it provided analogous results with the more elaborate multi-attribute utility model, showing that this easier to apply model can address effectively the stakeholder/user's preferences during the conceptual design phase. Both models identified the same aircraft configurations as dominant in terms of maximizing value or utility index while, in the surface plots, they both capture similar effects of design variables on the response, value or utility.

\subsection{Optimizing for User's and Manufacturer's Objectives}

The preferences of stakeholders other than the user were also implemented through the application of Game Theory. Two major stakeholders, user and manufacturer, were involved in a hybrid, cooperative/non-cooperative, non-zero sum, complete information, static game, modelling the interactions between their preferences and strategic choices, to accurately evaluate the alternative designs in the value driven conceptual design of the UAS.

For the selected strategic choices of the user (two scheduled replacement maintenance policies) and the manufacturer (two levels of reliability for aircraft components), there were four possible combinations. For each of them, a different NBS was identified, as the optimal design with the maximum value of the product of payoff functions of the two stakeholders. The four NBS generated from the cooperative nonzero sum, complete information games were used to form the non-cooperative, non-zero sum game and obtain the Nash equilibrium optimum design point.

In Table 5, the game is presented with the payoff values shown for all combinations of strategies. In each cell of the table, the values of the user's and manufacturer's payoff functions are presented for each NBS, obtained from the corresponding two players' cooperative (bargaining) non-zero sum game. The + and - 
signs represent the best move for each player in response to each move of the other player. For example, the - sign next to the value of 0.699 of the user's payoff function means that the component replacement maintenance policy is the best choice for the user (as opposed to 0.495 ), if the manufacturer selects to maintain the original reliability levels. In a similar manner, the + sign next to the value of 0.418 of the manufacturer's payoff function means that the original reliability level is the best response of the manufacturer (as opposed to 0.416), if the user selects the components' scheduled replacement policy. The cell that includes both + and - signs is a Nash equilibrium and a potential solution of the game, since it represents the optimal strategic choice of both players. This selection does not maximize the objective function of each individual player (user and manufacturer), but represents the optimal strategic choice in response to the other player's strategic choice, justified through successive elimination of strictly dominated strategies.

Table 5 User - Manufacturer Non-cooperative Game Near Here

In this case and based on the achieved values of payoff functions, the user will always choose a component scheduled replacement policy from the UAS replacement policy as a strictly dominating strategy, irrespectively of what the manufacturer selects. Hence, the manufacturer, knowing this fact, will select to maintain the components with the lower reliability levels. Thus, based on the specific preferences, a single Nash equilibrium was obtained: the Component Replacement Maintenance Policy and original (lower) reliability of critical components along with the corresponding values of design variables describing the optimum design. The optimal design obtained through the hybrid cooperative/non-cooperative game is a monolithic fuselage, $\mathrm{Y}$-shape tail, pusher propeller, UAS with a wing AR of approximately 12, wingspan of 
approximately $1.5 \mathrm{~m}$, battery capacity of $9.5-10 \mathrm{Ahr}$ and large scheduled components replacement intervals.

However, the conceptual design stage could also be modelled as a fully cooperative non-zero sum game solved as a pure bargaining problem. In this case, even the stakeholders' strategic choices are considered as mere design variables and the engineering design has an optimal solution based solely on the criterion of the maximization of the product of payoff functions. Hence, for the case of the UAS conceptual design, the optimal choices of the user and the manufacturer should be the UAS replacement policy and improved reliability of critical components, respectively. The corresponding optimal design would be a monolithic fuselage, Y-shape tail, pusher propeller UAS, with wing AR 12 , wing span of around $1.5 \mathrm{~m}$, battery capacity of $9.5-$ 10Ahr and large scheduled components replacement intervals.

\section{Discussion and Conclusions}

The main objective of this research is to develop a value model that employs successfully the value driven design philosophy in the conceptual design phase through a multi-stakeholder and multi-criteria decision making analysis. The multi-attribute value model is considered more appropriate to be used during the conceptual design phase to define objectively the set of optimal design alternatives, while the utility model should be employed as a second and more thorough approach in the later stages of engineering design, once the list of design alternatives is finalised. The value model, however, could be used in all phases of engineering design to define objectively the set of optimal design alternatives, frame and guide engineering design, provided that the stakeholder's preferences are updated based on information from simulation and prototyping. It is much more straightforward to apply, minimizing the interaction with 
the stakeholder/user, since ready to use value functions are automatically generated depending on their preferences. The evaluation process becomes more value focused, by identifying a priori needs and average levels of expectations of the user, than the alternative focused process of a classical multi-attribute utility model, with the stakeholder selecting the best from what is already available. AHP is also implemented in the weighting factors' computation to increase the accuracy and to assess the consistency of the stakeholders' responses. In addition, the deficiency of converting verbal preference responses between attributes to numerical values, using an arbitrary numerical scale in AHP for the calculation of weighting factors, was encountered and tackled with this value model. A synthesization averaging AHP-based method was introduced to deal successfully with the interpersonal preferential conflicts between individuals with the same objectives but different quantitative preferences, while maintaining the consistency.

The objectives of other than the user stakeholders with different interests/stakes were also taken into account in engineering design. Previously, Game Theory has been applied in engineering design as an optimization tool, modelling decision interactions among the stakeholders as players with either cooperative or non-cooperative games. This novel hybrid, cooperative/non-cooperative, non-zero sum and complete information game is capable of modelling the stakeholders' preferences, as well as capturing the interactions between their strategic choices, under the assumptions of players' instrumental rationality, common knowledge of rationality (CKR), consistently aligned beliefs $(\mathrm{CAB})$ and common knowledge of the game rules. Despite many objections expressed concerning the validity of these assumptions (Hargreaves Heap and Varoufakis 2004), Game Theory successfully employs the expected utility theory as the only acceptable exemplar of rational behaviour; it is used in this context to address 
the preferences of more than one stakeholder in this engineering design. This modelling of the optimal design alternative selection combines effectively the Nash bargaining solution (NBS), as the axiomatic based outcome of a hidden bargaining process, with the process of strategic interactions between the players of a non-cooperative game. Hence, the designer focuses not only on the articulation of the stakeholders' preferences but also on the interactions between their strategic choices, based on the information and their expectations concerning the other stakeholders' likely strategies.

The main objective of this research has been the development of a value model used in the identification of the optimal designs within a value driven design framework, addressing major preferences and risk attitudes of all stakeholders involved. The implementation of multi-objective and multi-stakeholder engineering design has been manifested and systematized; nevertheless, the selection of the appropriate value model depends on the current phase of engineering design process.

Current and future work includes the application of this methodology and framework in a practical case, capturing customer preferences/needs and trade-offs between them and assessing alternative designs, as well as exploring other applications with different players and strategic choices (such as performance requirements or manufacturing processes) within the value drive design framework. Further validation of the multi-attribute additive value model based on the results of the multi-attribute multiplicative utility model would also be beneficial. Experiments can also be carried out to validate the proposed non-zero sum, complete information game, based on the actual selections of decision makers involved in engineering design. Moreover, to capture the complexity of human decision making under uncertainty, biases, emotions and feelings of the individuals as decision makers, apart from rational behaviour, should be incorporated in engineering design. Thus, current work also includes the 
development of a value model, addressing the manifested deficiency of rationality assumption employed by classical decision making, with humans often violating the expected utility theory hypotheses and making irrational choices.

\section{Acknowledgements}

This work has been conducted through the Southampton University Engineering Centre of Excellence. The authors would like to thank the Defence Science and Technology Laboratory (DSTL) for the funding and support of this project. 


\section{References}

. "Isight \& the Simulia Execution Engine." from http://www.3ds.com/productsservices/simulia/portfolio/isight-simulia-execution-engine/overview/.

Abu Dabous, S., Alkass,S. (2010). "A Multi-attribute Ranking Method for Bridge

Management." Engineering Construction and Architectural Management 17(3): 282291.

Alexander, A. J. (1988). The Cost and Benefits of Reliability in Military Equipment. T. R. Corporation. Santa Monica, Ca, USA.

Annamdas, K. K. and S. S. Rao (2009). "Multi-objective optimization of Engineering Systems Using Game Theory and Particle Swarm Optimization." Engineering Optimization 41(8): 737-752.

Arrow, K. J. (1963). Social Choice and Individual Values. New Haven, CT, USA, Yale University Press.

Arsenyan, J., G. Buyukozkan and O. Feyzioglu (2015). "Modeling Collaboration Formation With a Game Theory Approach." Expert Systems with Applications 42: 2073-2085.

Berkhout, E. D., R. A. Schipper, H. Van Keulen and O. Coulibaly (2011).

"Heterogeneity in Farmers' Production Decisions and its Impact on Soil Nutrient use:

Results and Implications from Northern Nigeria." Agricultural Systems 104: 12.

Beygi, S., Haddad, O.B., Fallah-Mehdipour, E., Marino, M.A. (2014). "Bargaining Models for Optimal Design of Water Distribution Networks." Journal of Water Resources Planning and Management.

Butler, J., D. J. Morrice and P. W. Mullarkey (2001). "A Multiple Attribute Utility Theory Approach to Ranking and Selection." Management Science 47(6): 800-816. Carnero, M. C. (2013). "Multiciteria Model for Maintenance Benchmarking." Journal of Manufacturing Systems 33: 303-321.

Chang, Y. H. and C. H. Yeh (2001). "Evaluating airline competitiveness using multiattribute decision making." Omega 29: 405-415.

Chen, Y. M., Huang, P-N (2007). "Bi-Negotiation Integrated AHP in Suppliers Selection." International Journal of Operations and Production Management 27(11): 1254-1274.

Collopy, P. D. (2009). Aerospace System Value Models: A Survey and Observations. AIAA Space 2009 Conference \& Exposition. Pasadena, Ca, USA, AIAA. Collopy, P. D. and P. M. Hollingsworth (2011). "Value-Driven Design." Journal of Aircraft 48(3): 749-759.

Crossman, N. D., Bryan,B.A., King,D. (2011). "Contribution of Site Assessment Toward Prioritising Investment in Natural Capital." Environmental Modelling \& Software 26: 30-37.

Desideri, J.-A. (2012). "Cooperation and Competition in Multidisciplinary Optimization." Comput Optim Appl 52: 29-68.

Dijkstra, T. K. (2013). "On the Extraction of Weights from Pairwise Comparison Matrices." Central European Journal of Operations Research 21(1): 103-123.

Downen, T. D., D. J. Nightingale and C. L. Magee (2005). "Multi-Attribute Value Approach to Business Airplane Product Assessment." Journal of Aircraft 42(6): 13871395.

Dyer, J. S. (1990). "Remarks on the Analytic Hierarchy Process." Management Science 36(3): 249-258.

Dyer, J. S. and R. K. Sarin (1979). "Measurable Multiattribute Value Functions." Operations Research 27(4): 810-822. 
Dyer, R. F. and E. H. Forman (1992). "Group Decision Support with the Analytic Hierarchy Process." Decision Support Systems 52(2): 237-252.

Edwards, W. (2009). How to use multiattribute utility measurement for social decision making. New York, USA, Oxford University Press.

Edwards, W. and F. H. Barron (1994). "SMARTS and SMARTER: Improved Simple Methods for Multiattribute Utility Measurement." Organizational Behavior and Human Decision Processes 60(3): 306-325.

Elliot, M. A. (2010). "Selecting Numerical Scales for Pairwise Comparisons." Reliability Engineering and System Safety 95: 750-763.

Eres, M. H., M. Bertoni, M. Kossmann and J. P. Scanlan (2014). "Mapping Customer Needs to Engineering Characteristics: An Aerospace Perspective for Conceptual Design." Journal of Engineering Design 25(1-3): 64-87.

Feng, B. and F. Lai (2013). "Multi-attribute Group Decision Making With Aspirations: A Case Study." Omega 44: 136-147.

Fishburn, P. C. (1970). Utility Theory for Decision Making. New York, John Wiley \& Sons.

Gass, S. I. (2005). "The Great Debate: MAUT Versus AHP." Interfaces 35(4): 308-312. Gonzalez, L. F., J. Periaux, L. Damp and K. Srinivas (2007). "Evolutionary Methods for Multidisciplinary Optimization Applied to the Design of UAV Systems." Engineering Optimization 39(7): 773-795.

Habbal, A., J. Petersson and M. Thellner (2004). "Multidisciplinary Topology Optimization Solved as a Nash Game." International Journal of Numerical Methods in Engineering 61: 949-963.

Hargreaves Heap, S. P. and Y. Varoufakis (2004). Game Theory: A Critical Text. London \& New York, Routledge.

Harsanyi, J. C. (1995). "A New Theory of Equilibrium Selection for Games with Complete Information." Games and Economic Behavior 8: 91-122.

Harsanyi, J. C. and R. Selten (1972). "A Generalized Nash Solution for Two-person Bargaining Games with Incomplete Information." Management Science 18(5). Hu, Y. and S. S. Rao (2009). "Game-Theory Approach for Multiobjective Optimal Design of Stationary Flat-Plate Solar Collectors." Engineering Optimization 41(11): 1017-1035.

Huang, Y. S., W. C. Chang, W. H. Li and Z. L. Lin (2013). "Aggregation of Utility Based Individual Preferences for Group Decision Making." European Journal of Operational Research 229(2).

Hurley, W. J. and D. U. Lior (2002). "Combining Expert Judgment: On the Performance of Trimmed Mean Vote Aggregation Procedures in the Presence of Strategic Voting." European Journal of Operational Research 140: 142-147. Jiao, Y., M. Ma, Q. Yu, K. Yi and Y. Ma (2011). "Quality of Service Provisioning in Worldwide Interoperability for Microwave Access Networks Based on Cooperative Game Theory." IET Commun. 5(3): 284-295.

Keeney, R. L. (1996). Value-focused Thinking: a Path to Creative Decision Making. Cambridge, MA Harvard.

Keeney, R. L. and H. Raiffa (1976). Decisions with Multiple Objectives. Cambridge, UK, Press Syndicate of the Cambridge University.

Kim, S.-K. and O. Song (2009). "A MAUT Approach for Selecting a Dismantling Scenario for the Thermal Column in KRR-1." Annals of Nuclear Energy 36: 145-150. Kirkwood, C. W. and J. L. Corner (1993). "The effectiveness of partial information about attribute weights for ranking alternatives in multiattribute decision making." Organizational Behavior and Human Decision Processes 54(3): 456-476. 
Lai, V. S., B. K. Wong and W. Cheung (2002). "Group Decision Making in a multiple criteria environment: A case using the AHP in software selection." European Journal of Operational Research 137: 134-144.

Lee, D., Gonzalez, L.F., Periaux, J., Srinivas, K., Onate, E. (2011). "Hybrid-Game Strategies for Multi-objective Design Optimization in Engineering." Computers \& Fluids 47: 189-204.

Lootsma, F. A. (1991). Scale Sensitivity and Rank Preservation in a Multiplicative Variant of the AHP and SMART, Delft University of Technology.

Ma, K., Q. Han, C. Chen and X. Guan (2012). "Bandwidth Allocation for Cooperative Relay Networks Based on Nash Bargaining Solution." Int. J. Commun. Syst. 25: 10441058.

Matsatsinis, N., F., E. Grigoroudis and A. Samaras (2005). "Aggregation and Disaggregation of Preferences for Collective Decision-Making." Group Decision and Negotiation 14: 217-232.

Morera, O., Budescu, D. (1998). "A Psychometric Analysis of the "Divide and Conquer" Principle in Multicriteria Decision Making." Organizational Behavior and Human Decision Processes 75(3): 187-206.

Nash, J. F. (1950). "The Bargaining Problem." Econometrica 18(2): 155-162.

Nash, J. F. (1950). Non Co-operative Games. PhD, Princeton.

Papageorgiou, E., H. Eres and J. Scanlan (2015). Value Driven Conceptual Design of Unmanned Air System for Defense Applications. AIAA Aviation Conference 2015. Dallas, TX, USA.

Perez, J. (1995). "Some Comments on Saaty's AHP." Management Science 41(5): 10911095.

Poyhonen, M., Hamalainen, R.P. (2001). "On the Convergence of Multiattribute Weighting Methods." European Journal of Operational Research 129: 569-585. Roozenburg, N. F. M. and J. Eekels (1995). Product Design: Fundamentals and Methods. New York, Wiley.

Ross, A. M., M. G. O'Neill, D. E. Hastings and D. H. Rhodes (2010). Aligning Perspectives and Methods for Value-Driven Design. AIAA Space. AIAA. Anaheim, Ca, USA, AIAA.

Roy, B. (1991). "The Outranking Approach and the Foundations of ELECTRE methods." Theory and Decision 31(1): 49-73.

Runyan, P., W. Heping, W. Zhengping and L. Yu (2010). "Decision-making of Aircraft Optimum Configuration Utilizing Multi-Dimensional Game-Theory." Chinese Journal of Aeronautics 23: 194-197.

Saaty, T. L. (1980). The Analytic Hierarch Process. New York, USA, McGraw-Hill. Saaty, T. L. (2000). Fundamentals of Decision Making and Priority Theory With the Analytic Hierarchy Process

Saaty, T. L. and L. G. Vargas (2001). Models, Methods, Concepts and Applications of the Analytic Hierarchy Process. Masschusetts, USA, Kluwer Academic Publishers.

Salo, A. A. and R. P. Hamalainen (1997). "On the Measurement of Preferences in the Analytic Hierarchy Process." Journal of Multi-Criteria Decision Analysis 6: 309-319. Scholl, A., L. Manthey, R. Helm and M. Steiner (2005). "Solving Multiattribute Design Problems with Analytic Hierarchy Process and Conjoint Analysis: An Empirical Comparison." European Journal of Operational Research 164: 760-777.

Schuwirth, N., P. Reichert and J. Lienert (2012). "Methological Aspects of Multicriteria Decision Analysis for Policy Support: A Case Study on Pharmaceutical Removal from Hospital Wastewater." European Journal of Operational Research 220: 472-483. 
Semsar-Kazerooni, E. and K. Khorasani (2009). "Multi-agent Team Cooperation: a Game Theory Approach." Automatica 45: 2205-2213.

Sha, D. Y. and Z. H. Che (2005). "Virtual Integration with a MultiCriteria Partner Selection Model for the MultiEchelon Manufacturing System." International Journal of Advanced Manufacturing Technology 25: 793-802.

Sohn, K. Y., J. W. Yang and C. S. Kang (2001). "Assimilation of Public Opinions in Nuclear Decision Making Using Risk Perception." Annals of Nuclear Energy 28: 553563.

Tarek, Z. and M. Elsayed (2013). "Budget Allocation and Rehabilitation Plans for Water Systems Using Simulation Approach." Tunnelling and Underground Space Technology 36: 34-45.

Trappey, A., C. Trappey and W. C. Ni (2013). "A Multi-Agent Collaborative Maintenance Platform Applying Game Theory Negotiation Strategies." J Intell Manuf 24: 613-623.

Van Calker, K. J., P. B. M. Berentsen, C. Romero, G. W. J. Giesen and R. B. M. Huirne (2006). "Development and Application of a Multi-attribute Sustainability Function for Dutch Dairy Farming Systems." Ecological Economics 57: 640-658.

Von Neumann, J. and O. Morgenstern (1953). Theory of Games and Economic Behavior. Princeton,NJ, Princeton University Press.

Xiao, A., S. Zeng, J. K. Allen, D. W. Rosen and F. Mistree (2005). "Collaborative Multidisciplinary Decision Making Using Game Theory and Design Capability Indices." Research in Engineering Design 16: 57-72.

Xiao, M., Shao, X., Gao, L., Luo, Z. (2015). "A New Methodology for Multiobjective Multidisciplinary Design Optimization Problems Based on Game Theory." Expert Systems with Applications 42: 1602-1612.

Yaiche, H., R. R. Mazumdar and C. Roseberg (2000). "A Game Theoretic Framework for Bandwidth Allocation and Pricing in Broadband Networks." IEEE/ACM Transactions on Networking 8(5).

Yoon, K. P. and C. L. Hwang (1995). Multiple Attribute Decision Making. An Introduction. USA, Sage Publications Inc. 